\title{
A METHOD FOR INVESTIGATING GEOMETRIC PROPERTIES OF SUPPORT POINTS AND APPLICATIONS
}

\author{
BY
}

JOHNNY E. BROWN ${ }^{1}$

\begin{abstract}
A normalized univalent function $f$ is a support point of $S$ if there exists a continuous linear functional $L$ (which is nonconstant on $S$ ) for which $f$ maximizes $\operatorname{Re} L(g), g \in S$. For such functions it is known that $\Gamma=\mathbf{C}-f(U)$ is a single analytic arc that is part of a trajectory of a certain quadratic differential $Q(w) d w^{2}$. A method is developed which is used to study geometric properties of support points. This method depends on consideration of $\operatorname{Im}\left\{w^{2} Q(w)\right\}$ rather than the usual $\operatorname{Re}\left\{w^{2} Q(w)\right\}$. Qualitative, as well as quantitative, applications are obtained. Results related to the Bieberbach conjecture when the extremal functions have initial real coefficients are also obtained.
\end{abstract}

1. Introduction. Let $\nLeftarrow(U)$ denote the space of all functions analytic in the unit disk $U=\{z:|z|<1\}$. Given the topology of uniform convergence on compact subsets of $U$, the space $\mathcal{H}(U)$ becomes a locally convex topological vector space. A particular subset of $\mathcal{H}(U)$ is the class $S$. This class consists of all functions $f$ which are univalent in $U$ and normalized so that $f(0)=0$ and $f^{\prime}(0)=1$. We call $f \in S$ a support point if there exists a continuous linear functional $L$ defined on $\mathcal{H}(U)$ which is nonconstant on $S$ and

$$
\max _{g \in S} \operatorname{Re} L(g)=\operatorname{Re} L(f) .
$$

It is well known that all rotations of the Koebe function $k_{\theta}(z)=z /\left(1+e^{i \theta} z\right)^{2}$ are support points of $S$ as well as extreme points of the closed convex hull of $S[\mathbf{1}, \mathbf{1 4}]$. These functions map the unit disk onto the complement of a radial slit from $e^{-i \theta} / 4$ to infinity. A natural question to ask is which of the geometric properties of the functions $k_{\theta}$ are typical of those of arbitrary support points of $S$. It is known that if $f$ is a support point of $S$ then $\Gamma=\mathbf{C}-f(U)$ is a single analytic arc which tends to infinity with increasing modulus and $\Gamma$ possesses the $\pi / 4$-property: the angle between the radius and tangent vectors never exceeds $\pi / 4$ in absolute value $[\mathbf{9}, \mathbf{3}$, 6].

The principal tool used in the study of support points is the Schiffer variational method [12]. It implies that the arc $\Gamma$ satisfies a differential equation of the form

$$
w^{-2} L\left(f^{2} /(f-w)\right) d w^{2}>0 .
$$

In the past it was consideration of $\operatorname{Re}\left\{L\left(f^{2} /(f-w)\right)\right\}$ which led to geometric properties of $\Gamma$ (in particular, the $\pi / 4$-property is obtained in this way). The basic

Received by the editors September 13, 1982 and, in revised form, February 15, 1984; presented to the American Mathematical Society at the 88th annual meeting, January 1982.

1980 Mathematics Subject Classification. Primary 30C70; Secondary 30C50.

${ }^{1}$ This work was supported in part by NSF Grants MCS-8002703 and MCS-8200682. 
reason stems from the fact that for each $w \in \Gamma$, the competing function defined by $f_{w}(z)=w f(z) /(w-f(z))$ gives $L\left(f-f_{w}\right)=L\left(f^{2} /(f-w)\right)$. The purpose of this paper is to present a method whereupon consideration of $\operatorname{Im}\left\{L\left(f^{2} /(f-w)\right)\right\}$ will also lead to geometric properties of $\Gamma$. We apply this method to obtain qualitative, as well as quantitative, results about various support points. It is believed that the omitted arc $\Gamma$ of a support point has monotonic argument. We prove that this is indeed the case for the functional $L(g)=\alpha a_{2}+\beta a_{3}(\alpha, \beta \in \mathbf{C})$. This generalizes the result in $[\mathbf{4}]$. For this functional we also show that $\Gamma$ must lie in a certain halfplane that can be determined. A result is presented which implies the Bieberbach conjecture under a certain hypothesis.

2. A geometric method. The method presented in this section is implicit in work of Charzynski and Schiffer [5] and later in Bombieri [2]. We shall present the method in a form applicable to support points of $S$. This method is based on the behavior of trajectories of certain quadratic differentials. These properties can be found in $[\mathbf{7}$ and 10].

LEMMA 1. Let $\Omega$ be a simply-connected region not containing the origin and let $\Omega$ be bounded by a trajectory arc $\gamma$ of $\psi(\omega) d \omega^{2} / \omega^{2}$ and a simple arc $C$. Let $\gamma \cap C=\left\{p_{0}, p_{1}\right\}$ and suppose that $\psi / \omega^{2}$ is analytic on $\bar{\Omega} \backslash\left\{p_{0}, p_{1}\right\}$. Suppose further that $p_{0}$ and $p_{1}$ are not poles of order larger than one for $\psi(\omega) d \omega^{2} / \omega^{2}$. If $\psi \neq 0$ on $\partial \Omega \backslash\left\{p_{0}, p_{1}\right\}$ then there exists a simply-connected region $\Omega^{*} \subset \Omega$, bounded by a trajectory arc $\gamma^{*}$ of $\psi(\omega) d \omega^{2} / \omega^{2}$ and a connected subarc $C^{*} \subset C$, such that $\psi \neq 0$ on $\overline{\Omega^{*}}$.

PROOF. It is well known that there are exactly $n+2$ trajectories issuing from each zero of order $n$ of a quadratic differential and a single trajectory issuing at each simple pole $[\mathbf{1 0}]$. By our hypotheses we see that $\psi(\omega) d \omega^{2} / \omega^{2}$ has no poles in $\bar{\Omega} \backslash\left\{p_{0}, p_{1}\right\}$, and the points $p_{0}$ and $p_{1}$ are at worst simple poles. Hence, there are no trajectories in $\bar{\Omega}$ homotopic to a point since these occur only for poles of order larger than one $[\mathbf{1 0}]$.

Case $1 . \psi \neq 0$ in $\Omega$.

There are only finitely many trajectories issuing from $p_{0}$ and $p_{1}$. If $z^{*}$ is any fixed point of $\Omega$ not on these trajectories then there is a unique trajectory arc $\gamma^{*}$ in $\Omega$ passing through $z^{*}$. Now $\bar{\gamma}^{*}$ does not contain $p_{0}$ and $p_{1}$, and clearly, $\bar{\gamma}^{*} \cap C$ consists of exactly two points $\zeta_{1}$ and $\zeta_{2}$ (since no trajectory is homotopic to a point, and trajectories do not cross). Let $C^{*}$ be the connected subarc of $C$ from $\varsigma_{1}$ to $\varsigma_{2}$ and $\Omega^{*}$ the resulting simply-connected region with $\partial \Omega^{*}=\gamma^{*} \cup C^{*}$. This region satisfies the conclusion of the lemma.

Case 2. $\psi=0$ in $\Omega$.

There are at least three trajectories issuing from each of the finite number of zeros of $\psi$ in $\Omega$ and a finite number of trajectories from $p_{0}$ and $p_{1}$. Let $\tau$ denote the union of all such trajectories in $\Omega$, together with $\gamma$. Since there are no trajectories in $\bar{\Omega}$ homotopic to a point, each $\tilde{\gamma} \in \mathcal{T}$ has two endpoints which must be either $p_{0}, p_{1}$, a zero of $\psi$, or a point of $C$. Let $I=\bar{\tau} \cap C=\left\{\varsigma_{0}, \varsigma_{1}, \ldots, \varsigma_{n}\right\}$. This set is nonempty since $p_{0}, p_{1} \in I$. For convenience we let $\varsigma_{0}=p_{0}, \varsigma_{n}=p_{1}$, and we reindex if necessary so that as we traverse $C$ from $p_{0}$ to $p_{1}$ we follow $\varsigma_{0}, \varsigma_{1}, \ldots, \zeta_{n}$ in this order. (See Figure 1.) 


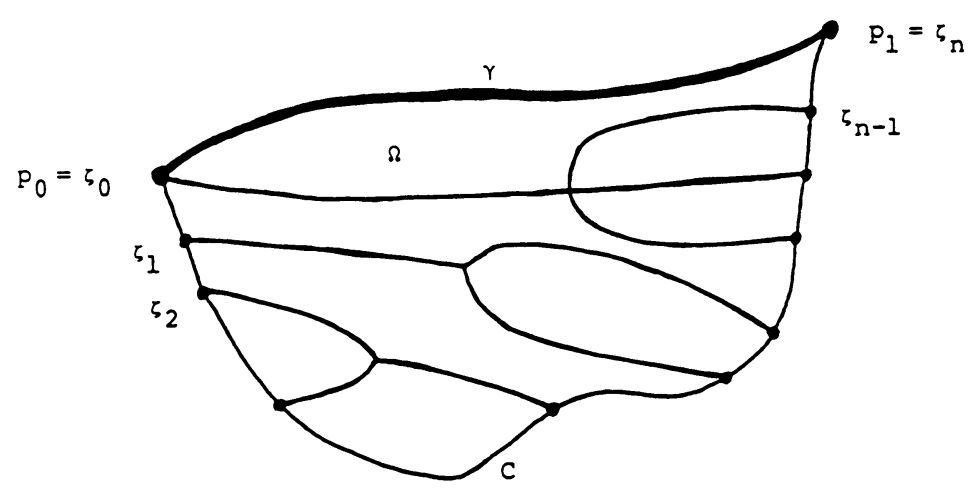

FIGURE 1

We let $I_{0}$ denote the set of all points of $I$ that can be joined to $\varsigma_{0}$ by a union of trajectories in $\tau$. Let $\zeta_{m_{0}}$ be the point of $I_{0}$ with $m_{0}$ minimal. Let $\Omega_{0} \subset \Omega$ be the resulting region bounded by the subarc $C_{0} \subset C$ from $\varsigma_{0}$ to $\zeta_{m_{0}}$ and the corresponding (unique) union of trajectories joining $\zeta_{0}$ to $\zeta_{m_{0}}$. If $\psi \neq 0$ in $\Omega_{0}$, we proceed as in Case 1 and we are done. If $\psi=0$ in $\Omega_{0}$, we let $I_{1}$ be the set of all points in $I$ that can be joined to $\zeta_{1}$ by a union of trajectories in $\tau$. Let $\zeta_{m_{1}}$ be the point of $I_{1}$ with $m_{1}$ minimal (clearly $m_{1}<m_{0}$ ). Let $\Omega_{1} \subset \Omega_{0}$ be the resulting region bounded by the subarc $C_{1} \subset C_{0}$ from $\zeta_{1}$ to $\zeta_{m_{1}}$ and the corresponding union of trajectories in $\tau$ joining $\zeta_{1}$ to $\zeta_{m_{1}}$. If $\psi \neq 0$ in $\Omega_{1}$, we proceed as in Case 1. If not, we continue this process, which terminates since $\psi$ has only a finite number of zeros in $\Omega$. The proof of the lemma is complete.

THEOREM 1. Let $\psi(\omega) d \omega^{2} / \omega^{2}$ be a quadratic differential which has a simple pole at $\omega=0$ and no other poles in $|\omega| \leq \rho$. Let $\Gamma_{0}$ be the unique trajectory which terminates at $\omega=0$. Suppose $\psi$ is nonzero on $\Gamma_{0}$ except at $\omega=0$.

(a) If $\operatorname{Im} \psi(\omega) \neq 0$ on the radial segment $J: \omega=t e^{i \theta}, 0<t<\rho$, then $\Gamma_{0} \cap J=\emptyset$.

(b) If $\operatorname{Im} \psi(\omega) \neq 0$ on the radial segment $J^{\prime}: \omega=t e^{i \theta}, 0 \leq \rho_{0}<t<\rho_{1}<\rho$, and $\Gamma_{0}$ lies in a sector of opening less than $2 \pi$, then $\Gamma_{0}$ intersects $\overline{J^{\prime}}$ (closure of $J^{\prime}$ ) at most once.

The condition $\operatorname{Im} \psi(\omega) \neq 0$ on $J$ says geometrically that no trajectory or orthogonal trajectory is ever tangent to $J$. In particular, if $\Gamma_{0}$ intersects $J$ it must actually cross $J$. Analytically the condition implies that $\operatorname{Im}\{\sqrt{\psi(\omega)}\} \neq 0$ on $J$; i.e., $\operatorname{Im}\{\sqrt{\psi(\omega)}\}$ retains its sign along $J$.

ProOF. (a) Assume that $\Gamma_{0} \cap J \neq \emptyset$. We would like to be able to apply Lemma 1 , so we first prove the existence of a simply-connected region $\Omega$ as in the lemma. Suppose first that there exists a point $\omega_{0} \in \Gamma_{0} \cap J$ nearest the origin. Let $C$ be that part of $J$ from 0 to $\omega_{0}$, and let $\gamma$ be that part of $\Gamma_{0}$ from 0 to $\omega_{0}$. Let $\Omega$ be the corresponding simply-connected region bounded by $\gamma$ and $C$. Assume now that $\Gamma_{0}$ crosses $J$ an infinite number of times near $\omega=0$. Since $\psi(\omega) d \omega^{2} / \omega^{2}$ has a simple pole at $\omega=0$, we know that $\Gamma_{0}$ is asymptotic to a line at $\omega=0$. Thus, the part of $\Gamma_{0}$ in a sufficiently small disk $|\omega|<\rho^{*}<\rho$ lies in a half-plane. In this disk we choose two consecutive points $\zeta_{1}, \zeta_{2}$ of $\Gamma_{0} \cap J$. Let $\gamma$ be the subarc of $\Gamma_{0}$ from $\zeta_{1}$ 
to $\zeta_{2}, C$ the part of $J$ from $\zeta_{1}$ to $\zeta_{2}$, and $\Omega$ the region bounded by $\gamma$ and $C$. Thus, in either case, we have found a region $\Omega$ as asserted.

For the choice of $\Omega$ as above we let $\gamma \cap C=\left\{p_{0}, p_{1}\right\}$ and observe that $\psi$ is analytic on $\bar{\Omega} \backslash\left\{p_{0}, p_{1}\right\}, \psi \neq 0$ on $\partial \Omega \backslash\left\{p_{0}, p_{1}\right\}$, and $p_{0}$ and $p_{1}$ are at worst simple poles of $\psi(\omega) d \omega^{2} / \omega^{2}$. We can thus apply Lemma 1 to conclude that there exists a region $\Omega^{*} \subset \Omega$ bounded by an arc $\gamma^{*}$ of a trajectory and a connected $\operatorname{arc} C^{*}$ of $J$ with $\psi$ nonzero on $\bar{\Omega}^{*}$. Suppose $C^{*}$ is the segment of $J$ from $\omega_{1}$ to $\omega_{2}$. Apply Cauchy's Theorem to conclude that $\int_{\partial \Omega^{*}} \sqrt{\psi(\omega)} d \omega / \omega=0$. Now since $\sqrt{\psi(\omega)} d \omega / \omega$ is real along $\gamma^{*}$. this implies that

$$
0=\operatorname{Im} \int_{\partial \Omega \Omega^{*}} \sqrt{\psi(\omega)} \frac{d \omega}{\omega}=\operatorname{Im} \int_{\omega_{1}}^{\omega_{2}} \sqrt{\psi(\omega)} \frac{d \omega}{\omega}=\int_{\omega_{1}}^{\omega_{2}} \operatorname{Im}\left\{\sqrt{\psi(\omega)} \frac{d \omega}{\omega}\right\} .
$$

However, as noted earlier, $\operatorname{Im} \sqrt{\psi(\omega)} \neq 0$ on $J$ and so $\int_{\omega_{1}}^{\omega_{2}} \operatorname{Im}\{\sqrt{\psi(\omega)} d \omega / \omega\} \neq 0$. This gives a contradiction and, hence, $\Gamma_{0} \cap J=\emptyset$.

(b) Assume $\Gamma_{0}$ meets $\overline{J^{\prime}}$ at least twice, say at $\omega_{1}$ and $\omega_{2}$. Now since $\Gamma_{0}$ lies in a sector of opening less than $2 \pi$, we let $\Omega$ be the region bounded by the subarc $\gamma$ of $\Gamma_{0}$ from $\omega_{1}$ to $\omega_{2}$ and by $C$, the part of $\overline{J^{\prime}}$ from $\omega_{1}$ to $\omega_{2}$. We apply the same argument as in (a) to arrive at a contradiction. This completes the proof of the theorem.

In view of the known properties of support points, this theorem is easily seen to be applicable. Let $f \in S$ be a support point for $L$ and let $\Gamma$ be its omitted arc. Then, by inverting $\omega=1 / w$, we see from (1) that $\Gamma_{0}=1 / \Gamma$ is a trajectory of the quadratic differential $\psi(\omega) d \omega^{2} / \omega^{2}$, where

$$
\psi(\omega)=L\left(\omega f^{2} /(\omega f-1)\right) .
$$

Schiffer $[\mathbf{1 3}]$ proved that $L\left(f^{2}\right) \neq 0$. Thus we see that $\psi(\omega) d \omega^{2} / \omega^{2}$ has a simple pole at $\omega=0$. Brickman and Wilken $[\mathbf{3}]$ have shown that $\psi$ is analytic on $\Gamma_{0}$. It is known that if $\psi$ vanishes on $\Gamma_{0}$, then $\Gamma_{0}$ must be radial and, hence, by the subordination principle, $f=k_{\theta}[\mathbf{1 4}]$. Thus, we may assume $\psi$ is nonzero on $\Gamma_{0}$. We now turn our attention to applications.

3. Applications. It is well known that if $\omega=0$ is a simple pole of $\psi(\omega) d \omega^{2} / \omega^{2}$, then precisely one trajectory and one orthogonal trajectory will terminate there $[\mathbf{1 0}$, p. 216]. Let $\Omega_{0}$ be a single trajectory arc in $|\omega| \leq \rho$ terminating at $\omega=0$. Since $d \omega^{2} / \omega^{2}>0$ holds for all radial lines, we see that if

$$
\operatorname{Im} \psi\left(t e^{i \theta_{1}}\right) \equiv \operatorname{Im} \psi\left(t e^{i \theta_{2}}\right) \equiv 0, \quad 0 \leq t \leq \rho,
$$

for distinct $\theta_{1}, \theta_{2} \in[0,2 \pi)$, then one of the radial segments $\omega=t e^{i \theta_{1}}$ or $\omega=$ $t e^{i \theta_{2}}, 0 \leq t \leq \rho$, must be a trajectory terminating at $\omega=0$. Suppose $R$ : $\omega=$ $t e^{i \theta_{1}}, 0 \leq t \leq \rho$, is a trajectory terminating at $\omega=0$. If $\psi$ is analytic on $\Gamma_{0}$ and nonzero on $\Gamma_{0} \backslash\{0\}$, then, because $\Gamma_{0}$ is a single analytic arc also terminating at $\omega=0$, we must have $\Gamma_{0}=R$.

THEOREM $<$. If $f(z)=z+\sum_{n=2}^{\infty} A_{n} z^{n}$ is a support point for the functional $L(g)=\alpha a_{2}+\beta a_{3}(\alpha, \beta \in \mathbf{C})$, and if $\Gamma$ is the arc omitted by $f$, then $\Gamma$ lies entirely in a half-plane and has monotonic argument.

PROOF. Clearly, if $\beta=0$ the only support points are $k_{\theta}$, so we may assume $\beta \neq 0$. We also invert by $\omega=1 / w$ and let $\Gamma_{0}=1 / \Gamma$. Thus, $\Gamma_{0}$ lies in $|\omega| \leq 4$ by 
the Koebe $\frac{1}{4}$-Theorem. It follows from (2) that $\Gamma_{0}$ is the trajectory (terminating at $\omega=0)$ of $\psi(\omega) d \omega^{2} / \omega^{2}$, where

$$
\psi(\omega)=-C \omega(1+D \omega)
$$

with $C=2 A_{2} \beta+\alpha$ and $D=\beta / C$. (Note that $L\left(f^{2}\right)=C \neq 0$.)

We first show that $\Gamma_{0}$ lies in a half-plane. Suppose $\operatorname{Im}\{D \bar{C}\} \neq 0$; then it is clear that $\operatorname{Im} \psi(\bar{C} t)=-|C|^{2} t^{2} \operatorname{Im}\{D \bar{C}\} \neq 0$ for $t \neq 0$. It is easy to check that all the hypotheses of Theorem 1 are satisfied, and from (a) we can conclude that $\Gamma_{0}$ lies entirely in a half-plane. In the case $\operatorname{Im}\{D \bar{C}\}=0$, we simply note that $\operatorname{Im} \psi(i \bar{C} t)=-|C|^{2} t \neq 0$ for $t \neq 0$. We apply Theorem $1(\mathrm{a})$ again to conclude that $\Gamma_{0}$ lies in a half-plane.

For each real $\theta$ we consider the radial segments in $|\omega| \leq 4$ defined by

$$
J_{\theta}: \omega=t e^{i \theta} / D, \quad 0<t \leq 4|D| .
$$

Then by putting $\theta_{0}=\arg (C / D)$ we see that

$$
\operatorname{Im} \psi\left(t e^{i \theta} / D\right)=-|C t / D|\left[\sin \left(\theta_{0}+\theta\right)+t \sin \left(2 \theta_{0}+\theta\right)\right] .
$$

If $\operatorname{Im} \psi\left(t e^{i \theta^{\prime}} / D\right) \equiv 0,0 \leq t \leq 4|D|$, for some $\theta^{\prime}$ we see from (3) that

$$
\operatorname{Im} \psi\left(t e^{i\left(\theta^{\prime}+\pi\right)} / D\right) \equiv 0, \quad 0 \leq t \leq 4|D|
$$

also holds. Now since $\omega=0$ is a simple pole of $\psi(\omega) d \omega^{2} / \omega^{2}$, by the above remarks we can conclude that $\Gamma_{0}$ is a radial segment. The subordination principle then yields $f=k_{\theta^{*}}$ for some real $\theta^{*}$. Hence, suppose $\operatorname{Im} \psi\left(t e^{i \theta} / D\right) \not \equiv 0,0 \leq t \leq 4|D|$, for all $\theta \in[0,2 \pi)$. Partition each $J_{\theta}$ at the zero of $\operatorname{Im} \psi\left(t e^{i \theta} / D\right)$. That is, let $J_{\theta}=L_{\theta} \cup \bar{l}_{\theta}$, where $L_{\theta}$ is the open segment of $J_{\theta}$ such that $\bar{L}_{\theta}$ contains the origin. (If $\operatorname{Im} \psi\left(t e^{i \theta} / D\right) \neq 0$ for $0<t \leq 4|D|$, we set $l_{\theta}=\left\{4 e^{i \theta}|D| / D\right\}$ and $L_{\theta}=J_{\theta} \backslash l_{\theta}$.) By construction, $\operatorname{Im} \psi(\omega)$ is nonzero on $L_{\theta}$ and $l_{\theta}$. We apply Theorem 1 (a) to each $L_{\theta}$ to conclude that $\Gamma_{0} \cap L_{\theta}=\emptyset$. Applying the second part of the theorem to each $\bar{l}_{\theta}$, we see that $\Gamma_{0}$ intersects $\bar{l}_{\theta}$ at most once. Hence, $\Gamma_{0}$ can intersect each radial segment in $|\omega| \leq 4$ at most once. This says that $\Gamma_{0}$, hence $\Gamma$, has monotonic argument. The proof of the theorem is complete.

Let us suppose that $f(z)=z+\sum_{n=2}^{\infty} A_{n} z^{n}$ belongs to $S$ and is a support point for $L(g)=a_{n}(n \geq 2)$. If we set $f(z)^{k}=\sum_{n=k}^{\infty} A_{n}^{(k)} z^{n}$, then the omitted arc $\Gamma=\mathbf{C}-f(U)$ satisfies

$$
-P_{n}\left(\frac{1}{w}\right)\left(\frac{d w}{w}\right)^{2}>0
$$

where

$$
P_{n}\left(\frac{1}{w}\right)=\sum_{k=1}^{n-1} \frac{A_{n}^{(k+1)}}{w^{k}} .
$$

In [8] it is shown that $A_{2} \neq 0$. If $A_{2}, \ldots, A_{n-1}$ are all real, then from (5) we see that $P_{n}$ is real on the real axis. The quadratic differential $-P_{n}(\omega) d \omega^{2} / \omega^{2}$ has a simple pole at $\omega=0$. Hence, the remark preceding Theorem 2 implies that $1 / \Gamma$ lies either on the positive or negative real axis. Hence, we must have $f(z)=z(1+z)^{2}$ or $f(z)=z /(1-z)^{2}$. This result can be improved. 
THEOREM 3. If $f(z)=z+\sum_{n=2}^{\infty} A_{n} z^{n} \in S$ is a support point for $L(g)=$ $a_{n}(n \geq 4)$ and $A_{2}, \ldots, A_{n-2}$ are real, then $f(z)=z /(1 \pm z)^{2}$, with $A_{n}=n$.

We shall make use of the following lemma.

LEMMA 2. If $f(z)=z+\sum_{n=2}^{\infty} A_{n} z^{n} \in S$ is a support point for $L(g)=a_{n}(n \geq$ 3) and $A_{n}^{(3)}, \ldots, A_{n}^{(n-1)}$ are all real, then $f(z)=z /(1 \pm z)^{2}$, with $A_{n}=n$.

Proof. Let $\Gamma=\mathbf{C}-f(U)$ be the omitted arc of $f$ and let $\Gamma_{0}=1 / \Gamma$. Thus, by (4), the arc $\Gamma_{0}$ satisfies $-P_{n}(\omega) d \omega^{2} / \omega^{2}>0$ in $|\omega|<4$. Since $A_{n}^{(3)}, \ldots, A_{n}^{(n-1)}$ are all real, it follows from (5) that

$$
\operatorname{Im}\left\{P_{n}(t)\right\}=t \operatorname{Im}\left\{A_{n}^{(2)}\right\}, \quad t \in \mathbf{R} .
$$

Assume that $\operatorname{Im}\left\{A_{n}^{(2)}\right\} \neq 0$. In this case we see that $\operatorname{Im}\left\{P_{n}(t)\right\} \neq 0$ along the real axis except at the origin. We apply Theorem $1(\mathrm{a})$ to conclude that $\Gamma_{0}$ meets the real axis only at $\omega=0$. In particular, $\Gamma_{0} \backslash\{0\}$ lies entirely in the upper or lower half-plane. We also know that

$$
-A_{2}=\frac{1}{2 \pi} \int_{0}^{2 \pi} \frac{1}{f\left(e^{i \theta}\right)} d \theta
$$

In other words, $-A_{2}$ lies in the closed convex hull of the point set $\Gamma_{0}$. Hence, $A_{2}$ lies in the upper or lower half-plane. However $A_{2}=A_{n}^{(n-1)} /(n-1)$ is real (and nonzero) and we arrive at a contradiction.

Thus, we must have $A_{n}^{(2)}$ real, and so $P_{n}$ is real on the real axis. We can then conclude from (4) that $\Gamma_{0}$ lies on the positive or negative real axis. Hence, $f(z)=z /(1 \pm z)^{2}$, with $A_{n}=n$.

PROOF OF THEOREM 3 . We first note that the formula

$$
A_{n}^{(m)}=\sum_{k=1}^{n-(m-1)} A_{k} A_{n-k}^{(m-1)}, \quad 2 \leq m \leq n,
$$

implies that $A_{n}^{(2)}=F_{2}\left(A_{2}, A_{3}, \ldots, A_{n-1}\right)$, where $F_{2}$ is a nonlinear function (with real cocfficients) of the $n-2$ variables indicated. Next we see that

$$
A_{n}^{(3)}=\sum_{k=1}^{n-2} A_{k} A_{n-k}^{(2)}=F_{3}\left(A_{2}, \ldots, A_{n-2}\right),
$$

where $F_{3}$ is a nonlinear function (with real coefficients) of the $n-3$ variables shown. Thus, in general, for $m=3,4, \ldots, n-1$ we see that

$$
A_{n}^{(m)}=F_{m}\left(A_{2}, \ldots, A_{n-m+1}\right)
$$

where $F_{m}$ has real coefficients and is a nonlinear function of the $n-m$ variables shown. In particular, the highest coefficient of $A_{k}$ appearing in $A_{n}^{(3)}, A_{n}^{(4)}, \ldots$, $A_{n}^{(n-1)}$ is clearly $A_{n-2}$. Hence, if $A_{2}, \ldots, A_{n-2}$ are real then $A_{n}^{(m)}$ is real for $m=3, \ldots, n-1$. Now apply Lemma 2 .

The author is indebted to the referees for their very helpful comments and suggestions. 


\section{REFERENCES}

1. L. Bieberbach, Über die Koeffizienten derjenigen Potenzreihen, welche eine schlichte Abbildung des Einheitskreises vermitteln, S.-B. Preuss. Akad. Wiss. (1916), 940-955.

2. E. Bombierei, A geometric approach to some coefficient inequalities for univalent functions, Ann. Scuola Norm. Sup. Pisa (3) 22 (1968), 377-397.

3. L. Brickman and D. Wilken, Support points of the set of univalent functions, Proc. Amer. Math. Soc. 42 (1974), 523-528.

4. J. E. Brown, Univalent functions maximizing $\operatorname{Re}\left\{a_{3}+\lambda a_{2}\right\}$, Illinois J. Math. 25 (1981), 446-454.

5. Z. Charzynski and M. Schiffer, A geometric proof of the Bieberbach conjecture for the fourth coeffi cient, Scripta Math. 25 (1960), 173-181.

6. P. L. Duren, Extreme points of spaces of univalent functions, Linear Spaces and Approximation, Birkhäuser-Verlag, Basel, 1978, pp. 471-477.

7. J. A. Jenkins, Univalent functions and conformal mapping, Springer-Verlag, Berlin, 1958.

8. W. Kirwan and R. Pell, Extremal problems for a class of slit conformal mapping, Michigan Math. J. 25 (1978), 223-232.

9. A. Pfluger, Lineare Extremalprobleme bei schlichten Funktionen, Ann. Acad. Sci. Fenn. Ser. A I no. 489 (1971).

10. Ch. Pommerenke, Univalent functions (with a chapter on quadratic differentials by G. Jensen), Vandenhoeck and Ruprecht, Göttingen, 1975.

11. A. C. Schaeffer and D. C. Spencer, Coefficient regions for schlicht functions, Amer. Math. Soc. Colloq. Publ., no. 35, Amer. Math. Soc., Providence, R.I., 1950.

12. M. Schiffer, A method of variation within the family of simple functions, Proc. London Math. Soc. (2) 44 (1938), 432-449.

13. __ On the coefficient problem for univalent functions, Trans. Amer. Math. Soc. 134 (1968), 95-101.

14. G. Schober, Univalent functions-selected topics, Lecture Notes in Math., vol. 478, SpringerVerlag, Berlin and New York, 1975.

Department of Mathematics, Purdue University, West lafayette, Indiana 47907 